\title{
About One Model Strategic Game of Collective Choice
}

\author{
Guram N. Beltadze \\ Faculty of Informatics and Control Systems, Georgian Technical University, Tbilisi, 0175, str. \\ Kostava 77 \\ Email:gbeltadze@yahoo.com \\ Jimsher A. Giorgobiani \\ N. Muskhelishvili Institute of Computational Mathematics, Georgian Technical University, \\ Tbilisi, 0175, str. Kostava 77 \\ Email: mzianachkebia@yahoo.com
}

\begin{abstract}
A model of dyadic non-cooperative game $\Gamma(H)$ is discussed in the paper for the set of one and the same players' strategies. The players make their choice sitting round the table and have the opportunity to coordinate only the meanings of utilities in every situation. Therefore the players' payoffs are given by $2 \times 2$ matrixes. A notion "the equalized situation" in mixed strategies which is at the same time the equilibrium is introduced. The theorem has been proved, which establishes the conditions of existance of an equalized situation in the given game. In the case of the existence algorithm is constructed. If equalized situation doesn't exist in the game, then there exists the equilibrium situation in the pure strategies and it is possible to find it by analysis of situations. $\Gamma(H)$ game's with bimatrix $\Gamma$ game in case of two players is given. The players' conditions of optimal mixed strategies existence in $\Gamma$ game is written. Relevant examples are solved and $\Gamma(H)$ game's application for finite amount of players' is discussed.
\end{abstract}

Index Terms - Game Theory, Non-cooperative Game, Dyadic Game, Strategy, Collective choice, Equilibrium Situation.

\section{Introduction}

Mathematical methods in the normative theory of making decisions are used for two purposes: for clearing the existing reality and for estabishment of its rational behaviour. In the process of making collective choice the new fundamental problems are rising. In the last period of time the problem of democracy has got a lot of interest in social - economic and political sciences and the question is if we can define the correct methods of individual decissions collection in order to get a correct collective choise.

Every collective choice (decission) mainly takes place in the case of different interest of individuals. In this case the process of collective choice is a game. In that case if they can not come to an agreement on choosing strategies (variants, alternatives) the process of making collective choise is a non-cooperative game.

Game theory is a modern field of making decisions [6]. For the time being, non-cooperative, e.i. strategic games for making collective choise strategic modelling is taking place not only in economics, business and management, but in many other fields of science and life. For example, in social field [3], in politics [1,7,8,9], in security [2, 5], in information theory [10] and others.

Suppose there is a collective that consists of $n$ individuals $N=\{1,2, \ldots, n\}$. Choosing the only element from the set of $A=\left\{a_{1}, a_{2}, \ldots, a_{m}\right\}$ strategies is their task of collective choice. In a lot of process of social, economic and political situations the collective choice is led to the choice of one of them from two possible strategies. We're dicussing a case like this and mean that the set of each individ strategies of the collective is the same and it has got the following form $A_{i}=\{1,2\}, i \in N$. Here the situation in the pure strategies has got the following form $x=\left\{x_{1}, x_{2}, \ldots, x_{n}\right\}$, where $x_{i} \in\{1,2\}$ and $H_{i}$ is the $i \in N$ player's utility function. In this case noncooperative game 


$$
\Gamma=<N,\left\{A_{i}\right\}_{i \in N},\left\{H_{i}\right\}_{i \in N}>,
$$

where $H_{i}: \prod_{i \in N} A_{i} \rightarrow R$ is called a dyadic scalar game.

Dyadic games is a significant class of noncooperative games, where a player has two strategies. In the case of two players one of the well-known game is for example the "Prisoner's Dilemma". They are used for modelling simple situations taking from the socialpolitical life, where the elements of competition, coordination and cooperation participate in the form of different variants: vote or not, confess or not, sign or not and etc.

Non-cooperative dyadic games have a certain place in the books and articles published in the following fields of study: Game Theory, Biology, Psychology and so on.

In the case of scalar utilities, dyadic games arc described in detail for example Vorob'ev's book [12]. In such games, the conditions of Nash's equilibrium existing in a completely mixed strategies are given by analytical relations.

In this paper, special class of dyadic games is discussed, that is to say. we assume that we have a finite set of players $N=\{1,2, \ldots, n\}$ sitting at a round table. Each of them has two strategies. Let us discuss the situation when each $i=1,2, \ldots, n$ players' utility (payoff') depends on own estrategie and $i+1$-player's strategies (taking $n+1=1$ ) and they can agree with each other in each situation only about the meanings of payoffs. In this case it is natural to consider that the player's payoff functions have the following form by matrix:

$$
H_{1}\left(x_{1}, x_{2}\right), H\left(x_{2}, x_{3}\right), \ldots, H_{n}\left(x_{n}, x_{1}\right) .
$$

Hence, we have got strategic $\Gamma$ game with matrix payoffs $H_{1}, H_{2}, \ldots, H_{n}$ and we note by $\Gamma(H)$, where each player's first strategy is 1 , the second strategy is 2 .

\section{The main part}

In (1) kind of non-cooperative $\Gamma(H)$ game with payoffs we determined the conditions of the existence the equilibrium situation in mixed strategies. With the help of such conditions in the case of $n=2$ player's case the equivalency of $\Gamma(H)$ game's solution is studied related to the analysis of common $2 \times 2$ bimatrix game, which is given by algebraic form. But generally the analysis of $2 \times 2$ bimatrix game is held by graphical form [12]. The focus is on finding the equilibrium situation in pure strategies in case of two or more players' in $\Gamma(H)$ game. $\Gamma(H)$ game's model we have used for lexicographic games [11]. In the present article using the model of $\Gamma(H)$ game is studied in an organization of a teaching process. Relevant examples are discussed.

Obviously, in the case of payoffs given by (1), $\Gamma(H)$ game is a dyadic non-cooperative game, in which each player's payoff is given by means of a $2 \times 2$ matrix. For $n=2$ player's $\Gamma(H)$ has the following form:

$$
\begin{gathered}
H_{1}\left(x_{1}, x_{2}\right)=\left(\begin{array}{ll}
a_{11} & a_{12} \\
a_{21} & a_{22}
\end{array}\right) \\
H_{2}\left(x_{2}, x_{1}\right)=\left(\begin{array}{ll}
b_{11} & b_{12} \\
b_{21} & b_{22}
\end{array}\right) .
\end{gathered}
$$

In this case game (3) is the same as the following bimatrix game

$$
\Gamma=\left(\begin{array}{ll}
\left(a_{11}, b_{11}\right) & \left(a_{12}, b_{21}\right) \\
\left(a_{21}, b_{12}\right) & \left(a_{22}, b_{22}\right)
\end{array}\right)
$$

Therefore above stated "Prisoner's Dilemma" game with strategies $A_{1}=A_{2}=\{1$-Non Confess, 2 -Confess $\}$

$$
\Gamma=\left(\begin{array}{ll}
(-1,-1) & (-10,0) \\
(0,-10) & (-8,-8)
\end{array}\right)
$$

is the same as the following $\Gamma(H)$

$$
H_{1}=\left(\begin{array}{cc}
-1 & -10 \\
0 & -8
\end{array}\right), H_{2}=\left(\begin{array}{cc}
-1 & -10 \\
0 & -8
\end{array}\right) \text {. }
$$

Note for players' $N=\{1,2, \ldots, n\}$ in $\Gamma(H)$ game $X_{i}=\left(p_{i}, 1-p_{i}\right)$ is the mixed strategy of $i \in N$ player, where $p_{i}$ is first strategy's choosing probability. The situation in the mixed strategies will be $X=\left(X_{1}, X_{2}, \ldots, X_{n}\right)$.

Definition. The situation in mixed strategies $\mathrm{X}=\left(\mathrm{X}_{1}, \mathrm{X}_{2}, \ldots, \mathrm{X}_{\mathrm{n}}\right)$ in $\Gamma(H)$ game is called the equilibrium situation, if

$H_{i}(X) \geq H_{i}\left(X \mathrm{II}_{\mathrm{i}} X_{i}\right)$ for any $i \in N$, and for any $x_{i} \in\{1,2\}$.

The situation $X=\left(X_{1}, X_{2}, \ldots, X_{n}\right)$ in $\Gamma(H)$ game is called equalized sitiation, if for any $i \in N$ the folloving equation takes plase:

$$
H_{i}\left(X \mathrm{II}_{\mathrm{i}} 1\right)=H_{i}\left(X \mathrm{II}_{\mathrm{i}} 2\right),(7)
$$

where $X \mathrm{II}_{\mathrm{i}} \mathrm{k}=\left(X_{1}, \ldots, X_{k-1}, k, X_{k+1}, \ldots, X_{n}\right)$.

According to the definition in $\Gamma(H)$ game the equalized situation is the equilibrium situation in the same game. At the same time, because of the situation $X=\left(X_{1}, X_{2}, \ldots, X_{n}\right)$ in completely mixed strategies 
(when every $\left.0<p_{i}<1, i=1, \ldots, n\right)$ in $\Gamma(H)$ game be equalized, it is necessary and sufficient that for every $i \in N$ the equation (7) should be taken place (Borov'ev [12]). Therefore, the rational behind the desire and tendency of the players is to attain equalized situations, since a tendency towards equalized situation can be viewed as a kind of optimal behavior.

Let the following equation be used for $i \in N$ : $\Delta x_{i} H_{i}\left(x_{i+1}\right)=H_{i}\left(2, x_{i+1}\right)-H_{i}\left(1, x_{i+1}\right)$,

$$
\begin{aligned}
\Delta x_{i}, & x_{i+1} H_{i}=\Delta x_{i} H_{i}(2)-\Delta x_{i} H_{i}(1)= \\
& =H_{i}(1,1)+H_{i}(2,2)-H_{i}(2,1)-H_{i}(1,2),
\end{aligned}
$$

where $i \in N$ and the sum $\mathrm{i}+1$ acquired by $n$-modulo.

Theorem. Dyadic $\Gamma(H)$ game with payoff matrix has an equalized equilibrium situation in mixed strategies if and only if

$$
F\left(x_{i}\right) \equiv \Delta x_{i} H_{i}(1) . \Delta x_{i} H_{i}(2) \leq 0, i \in N .
$$

Moreover, the mixed strategy of the player $X_{i}=\left(p_{i}, 1-p_{i}\right), i \in N$ in the equalized situation is defined in the folloving way:

(i) if $\quad \Delta x_{i} H_{i}(1) \cdot \Delta x_{i} H_{i}(2) \leq 0 \quad$ and $\Delta x_{i} H_{i}(1) \neq \Delta x_{i} H_{i}(2)$, then

$$
p_{i+1}=\frac{\Delta x_{i} H_{i}(2)}{\Delta x_{i}, x_{i+1} H_{i}} \text {; }
$$

(ii) if $\Delta x_{i} H_{i}(1)=\Delta x_{i} H_{i}(2)=0, \quad$ then $p_{i+1} \in[0,1]$.

In other case in $\Gamma(H)$ game there exists an equilibrium situation in pure strategies.

Proof. According the to definition the situation $X=\left(X_{1}, X_{2}, \ldots, X_{n}\right)$ in $\Gamma(H)$ game is equalized if and only if the following equalities takes place:

$$
\begin{gathered}
H_{i}(1,1) p_{i+1}+H_{i}(1,2)\left(1-p_{i+1}\right)=H_{i}(2,1) p_{i+1}+ \\
H_{i}(2,2)\left(1-p_{i+1}\right), \quad i=1,2, \ldots, n .
\end{gathered}
$$

Hence one obtains

$$
p_{i+1} \cdot \Delta x_{i}, x_{i+1} H_{i}=\Delta x_{i} H_{i}(2) \text {. }
$$

Therefore, for the existence of an equalized situation in $\Gamma(H)$ game it is necessary and sufficient to fullfil one of these two conditions

$$
\Delta x_{i}, x_{i+1} H_{i} \neq 0,0 \leq \frac{\Delta x_{i} H_{i}(2)}{\Delta x_{i}, x_{i+1} H_{i}} \leq 1
$$

or

$$
\Delta x_{i}, x_{i+1} H_{i}=\Delta x_{i} H_{i}(2)=0 \text {. (9) }
$$

From these conditions, (8) is equal to the following inequalities:

$$
\begin{gathered}
\Delta x_{i} H_{i}(1) \cdot \Delta x_{i} H_{i}(2) \leq 0 \text { and } \\
\Delta x_{i} H_{i}(1) \neq \Delta x_{i} H_{i}(2),
\end{gathered}
$$

but condition (9) is expressed in such way that

$$
\Delta x_{i} H_{i}(1)=\Delta x_{i} H_{i}(2)=0 \text {. }
$$

In fact, let

$$
\Delta x_{i}, x_{i+1} H_{i}=\Delta x_{i} H_{i}(2)-\Delta x_{i} H_{i}(1)>0 .
$$

Then from (8) we get

$$
\Delta x_{i} H_{i}(2) \leq \Delta x_{i} H_{i}(2)-\Delta x_{i} H_{i}(1)
$$

and $\quad \Delta x_{i} H_{i}(1) \leq 0$. As in (8) $\Delta x_{i} H_{i}(2) \geq 0$, therefore

$$
\Delta x_{i} H_{i}(1) . \Delta x_{i} H_{i}(2) \leq 0 .
$$

If $\Delta x_{i}, x_{i+1} H_{i}<0$, then from (3) we get $\Delta x_{i} H_{i}(1) \geq 0 . \quad$ As in (3) $\Delta x_{i} H_{i}(2) \leq 0$, therefore

$$
\Delta x_{i} H_{i}(1) . \Delta x_{i} H_{i}(2) \leq 0 .
$$

Now prove on the contrary. Let

$$
\Delta x_{i} H_{i}(1) \cdot \Delta x_{i} H_{i}(2) \leq 0
$$

and

$$
\Delta x_{i} H_{i}(1) \neq \Delta x_{i} H_{i}(2) .
$$

If here $\Delta x_{i} H_{i}(1) \geq 0$, then $\Delta x_{i} H_{i}(2) \leq 0$.

Therefore $\Delta x_{i}, x_{i+1} H_{i}<0$ and

$$
0 \leq \frac{\Delta x_{i} H_{i}(2)}{\Delta x_{i}, x_{i+1} H_{i}} .
$$

Let show, that this fraction is not more than 1 . Let us suppose that it is more than 1 :

$$
\frac{\Delta x_{i} H_{i}(2)}{\Delta x_{i}, x_{i+1} H_{i}}>1 \text {. }
$$

According to that fraction

$$
\Delta x_{i} H_{i}(2)<\Delta x_{i} x_{i+1} H_{i} \text { or } \Delta x_{i} H_{i}(1)<0 \text {. }
$$

This is controdiction to the assumption $\Delta x_{i} H_{i}(1) \geq 0$. So, when $\Delta x_{i} H_{i}(1) \geq 0$, we obtain

$$
0 \leq \frac{\Delta x_{i} H_{i}(2)}{\Delta x_{i}, x_{i+1} H_{i}} \leq 1 .
$$

We'll obtain the same when $\Delta x_{i} H_{i}(1) \leq 0$ and thus the (8) is fullfilled.

Hence, according to our conditions, in the first case one gets

$$
p_{i+1}=\frac{\Delta x_{i} H_{i}(2)}{\Delta x_{i}, x_{i+1} H_{i}}
$$

and in the second case $p_{i+1}$ is any number from $[0,1]$. 


\section{Practical part}

At first let discuss the following examples particulary two players' dyadic $\Gamma(H)$ game with matrixes of payoff. Let, write the necessary formulae for analysis of (3) game. As we see, this game is different from the ordinary non-cooperative game - bimatrix $\Gamma$ game (4). $\Gamma(H)$ game's in $H_{1}$ player $i=1$ chooses the line but $i=2$ player chhoses the column. In $H_{2}$ $i=2$ player chooses the line, $i=1$ player chooses the column.

According to our notes for (3) $\Gamma(H)$ game we write:

$$
\begin{gathered}
F\left(x_{i}\right) \equiv \Delta x_{i} H_{i}(1) . \Delta x_{i} H_{i}(2)= \\
\left(H_{i}(2,1)-H_{i}(1,1)\right) \cdot\left(H_{i}(2,2)-H_{i}(1,2)\right), i=1,2 ; \\
\Delta x_{i}, x_{i+1} H_{i}=H_{i}(1,1)+H_{i}(2,2)-H_{i}(2,1)-H_{i}(1,2), \\
i=1,2 ; \\
p_{1}=\frac{H_{2}(2,2)-H_{2}(1,2)}{H_{2}(1,1)+H_{2}(2,2)-H_{2}(2,1)-H_{2}(1,2)} \\
p_{2}=\frac{H_{1}(2,2)-H_{1}(1,2)}{H_{1}(1,1)+H_{1}(2,2)-H_{1}(2,1)-H_{1}(1,2)}
\end{gathered}
$$

Example 1. $\Gamma(H)$ game of two players' has got the folloving form

$$
H_{1}=\left(\begin{array}{ll}
1 & 2 \\
3 & 1
\end{array}\right), H_{2}=\left(\begin{array}{cc}
0 & 1 \\
1 & -2
\end{array}\right)
$$

Here

$$
\begin{aligned}
& F\left(x_{1}\right)=(3-1) \cdot(1-2)=-2<0, \\
& F\left(x_{2}\right)=(1-0) \cdot(-2-1)=-3<0 .
\end{aligned}
$$

Therefore in the given game there exists the equilibrium situation in the mixed strategies and

$$
p_{1}=\frac{-2-1}{0-2-1-1}=\frac{3}{4}, \quad p_{2}=\frac{1-2}{0-2-1-1}=\frac{1}{3} \text {. }
$$

Hence, in the given game $i=1$ the player's optimal mixed strategy is $X_{1}^{*}=(3 / 4,1 / 4)$, but $i=2$ the player's optimal mixed strategy is $X_{2}^{*}=(1 / 3,2 / 3)$ and in the given dyadic $\Gamma(H)$ game the equalized and therefore the equilibrium situation is $X^{*}=((3 / 4,1 / 4),(1 / 3,2 / 3))$.

In the given game there also exists the equilibrium situation in pure strategies $X^{* *}=(2,1)$. This means, thet in $H_{1}$ player $i=1$ chooses the second line and $i=2$ player chooses the first column, but in the $H_{2}$ player $i=2$ chooses the first line, $i=1$
- the second column.

Example 2. For $\Gamma(H)$ (6) game we have

$$
\begin{aligned}
& F\left(x_{1}\right)=(0+1) \cdot(-8+10)=2>0, \\
& F\left(x_{2}\right)=(0+1) \cdot(-8+10)=2>0
\end{aligned}
$$

and therefore in (6) game or (5) game there is no equilibrium situation in the mixed strategies. There exists the only equilibrium situation in the pure strategies $X^{* *}=(2,2)$.

Example 3. Let us discuss a four person's dyadic $\Gamma(H)$ game with the following payoff matrices

$$
\begin{gathered}
H_{1}=\left(\begin{array}{cc}
1 & -1 \\
0 & 1
\end{array}\right), H_{2}=\left(\begin{array}{cc}
0 & 1 \\
2 & -1
\end{array}\right), \\
H_{3}=\left(\begin{array}{cc}
3 & 2 \\
-2 & 1
\end{array}\right), H_{4}=\left(\begin{array}{cc}
-1 & 1 \\
0 & -1
\end{array}\right) .
\end{gathered}
$$

Let us discuss whether there exists or not an equalized equilibrium situation in the given game. Let us verify the conditions of Theorem for every $i=1,2,3,4$. Note that

$$
\begin{gathered}
F\left(x_{i}\right)=\Delta x_{i} H_{i}(1) . \Delta x_{i} H_{i}(2)= \\
=\left(H_{i}(2,1)-H_{i}(1,1)\right) \cdot\left(H_{i}(2,2)-H_{i}(1,2) .\right.
\end{gathered}
$$

The meanings of $F\left(x_{i}\right)$ are:

$$
\begin{gathered}
F\left(x_{1}\right)=(-1) \cdot 2<0, F\left(x_{2}\right)=2 \cdot(-2)<0, \\
F\left(x_{3}\right)=(-5) \cdot(-1)>0, F\left(x_{4}\right)=1 \cdot(-2)<0 .
\end{gathered}
$$

Here for $i=3$ the condition of Theorem $F\left(x_{3}\right) \leq 0$ is not fulfilled. Thus in the given $\Gamma(H)$ game there is no equalized situation or equilibrium situation in the mixed strategies. Therefore in the given $\Gamma(H)$ game there exists the equilibrium situation in the pure strategies. By analizing all the situation, which amount is 16 , we find that $X^{*}=(2,2,1,1)$ is the equilibrium situation in $\Gamma(H)$ game in pure strategies.

The situation $X^{*}=(2,2,1,1)$ in the given game can be described as the following way: in $H_{1}$ player $i=1$ chooses the second strategy (the second line), $i=2$ player chooses the second strategy (the second column); in $H_{2}$ player $i=2$ chooses the second strategy (the second line), $i=3$ - the first strategy (the second column); in $H_{3}$ player $i=3$ chooses the first strategy, $i=4$ - the first strategy; in $\mathrm{H}_{4}$ $i=4$ chooses the first strategy and $i=1$ - the second strategy. Therefore in $\Gamma(H)$ game players' payoffs are 
$v_{1}=1, v_{2}=2, v_{3}=3, v_{4}=1$.

According to the results of the 3 - nd and 4 - th players agreement. Let

$$
H_{3}=\left(\begin{array}{cc}
3 & 1 \\
-2 & 2
\end{array}\right) \text {. }
$$

Then $F\left(x_{3}\right)=(-5) \cdot 1<0$. Let's note the obtained game by $\Gamma_{1}(H)$.

Therefore, the condition of Theorem $F\left(x_{i}\right)<0, i=1,2,3,4$ has been fulfilled and there is an equalized situation in this game.

Now, let us find the mixed strategy of each player in the equalized situation. According to the formula, we can write

$$
\begin{gathered}
p_{1}=\frac{\Delta x_{4} H_{4}(2)}{\Delta x_{4}, x_{1} H_{4}}=\frac{-2}{-3}=\frac{2}{3}, X_{1}^{*}=(2 / 3,1 / 3), \\
p_{2}=\frac{\Delta x_{1} H_{1}(2)}{\Delta x_{1}, x_{2} H_{1}}=\frac{2}{3}, X_{2}^{*}=(2 / 3,1 / 3), \\
p_{3}=\frac{\Delta x_{2} H_{2}(2)}{\Delta x_{2}, x_{3} H_{2}}=\frac{-2}{-4}=\frac{1}{2}, X_{3}^{*}=(1 / 2,1 / 2), \\
p_{4}=\frac{\Delta x_{3} H_{3}(2)}{\Delta x_{3}, x_{4} H_{3}}=\frac{1}{6}, X_{4}^{*}=(1 / 6,5 / 6) .
\end{gathered}
$$

In this dyadic $\Gamma_{1}(H)$ game, the equalized and therefore the equilibrium situation is the following:

$X^{*}=((2 / 3,1 / 3),(2 / 3,1 / 3)$,

$$
(1 / 2,1 / 2),(1 / 6,5 / 6)) \text {. }
$$

In $\Gamma_{1}(H)$ game the players' payoffs $v_{i}(i=1,2,3,4)$ are equal to:

$$
\begin{aligned}
& v_{1}=X_{1} H_{1} X_{2}^{T}=(2 / 3,1 / 3)\left(\begin{array}{cc}
1 & -1 \\
0 & 1
\end{array}\right)(2 / 3,1 / 3)^{T}=\frac{1}{3}, \\
& v_{2}=X_{2} H_{2} X_{3}^{T}=(2 / 3,1 / 3)\left(\begin{array}{cc}
0 & 1 \\
2 & -1
\end{array}\right)(1 / 2,1 / 2)^{T}=\frac{1}{2}, \\
& v_{3}=X_{3} H_{3} X_{4}^{T}=(1 / 2,1 / 2)\left(\begin{array}{cc}
3 & 1 \\
-2 & 2
\end{array}\right)(1 / 6,5 / 6)^{T}=\frac{4}{3}, \\
& v_{4}=X_{4} H_{4} X_{1}^{T}=(1 / 6,5 / 6)\left(\begin{array}{cc}
-1 & 1 \\
0 & -1
\end{array}\right)(2 / 3,1 / 3)^{T}= \\
& =-\frac{1}{3} .
\end{aligned}
$$

In the same $\Gamma_{1}(H)$ game there as well exists the equilibrium situation in pure strategies $X^{* *}=(2,2,1,1)$, where the players' payoffs are relatively $v_{1}=1, v_{2}=2, v_{3}=3, v_{4}=1$.

The equalized situation in $\Gamma(H)$ game may exist no only in completely mixed strategies. We shal show it in the following example.

Example 4. Let us consider the dyadic $\Gamma(H)$ game of three players with the following payoff matrices

$$
H_{1}=\left(\begin{array}{ll}
2 & 1 \\
2 & 1
\end{array}\right), H_{2}=\left(\begin{array}{ll}
4 & 5 \\
2 & 6
\end{array}\right), H_{3}=\left(\begin{array}{ll}
0 & 3 \\
1 & 2
\end{array}\right) .
$$

Here

$$
\begin{gathered}
F\left(x_{1}\right)=0 \cdot 0=0, \quad F\left(x_{2}\right)=(-2) \cdot 1<0, \\
F\left(x_{3}\right)=1 \cdot(-1)<0, \\
\Delta x_{1}, x_{2} H_{1}=0, \quad \Delta x_{2}, x_{3} H_{2}=3, \\
\Delta x_{3}, x_{1} H_{3}=-2, \\
\Delta x_{1} H_{1}(2)=1-1=0, \quad \Delta x_{2} H_{2}(2)=6-5=1, \\
\Delta x_{3} H_{3}(2)=2-3=-1 .
\end{gathered}
$$

According to Theorem we obtain

$$
\begin{array}{ll}
p_{1}=\frac{\Delta x_{3} H_{3}(2)}{\Delta x_{3}, x_{1} H_{3}}=\frac{1}{2}, & X_{1}^{*}=(1 / 2,1 / 2), \\
p_{2} \in[0,1], & X_{2}^{*}=(p, 1-p), \\
p_{3}=\frac{\Delta x_{2} H_{2}(2)}{\Delta x_{2}, x_{3} H_{2}}=\frac{1}{3}, & X_{3}^{*}=(1 / 3,2 / 3) .
\end{array}
$$

In $\Gamma(H)$ game the equalized situation is the following:

$$
X^{*}=((1 / 2,1 / 2),(p, 1-p),(1 / 3,2 / 3)) .
$$

The players' payoffs $v_{i}(i=1,2,3)$ are equal to:

$$
\begin{aligned}
v_{1}=X_{1} H_{1} X_{2}^{T} & =p+1, v_{2}=X_{2} H_{2} X_{3}^{T}=\frac{14}{3}, \\
v_{3} & =X_{3} H_{3} X_{1}^{T}=\frac{3}{2} .
\end{aligned}
$$

Example 5. In the dyadic scalar $\Gamma(H)$ game of three players with payoff matrix

$$
H_{1}=\left(\begin{array}{ll}
2 & 1 \\
3 & 0
\end{array}\right), H_{2}=\left(\begin{array}{ll}
2 & 0 \\
3 & 4
\end{array}\right), H_{3}=\left(\begin{array}{ll}
2 & 3 \\
0 & 2
\end{array}\right)
$$


we have $F\left(x_{1}\right)=1 \cdot(-1)<0, \quad F\left(x_{2}\right)=1 \cdot 4>0$, $F\left(x_{3}\right)=(-2) \cdot(-1)>0$. Hence the conditions of Theorem are not fulfilled. The equilibrium situation in pure strategies exists in game

$$
X^{*}=((1,0) \equiv 1,(0,1) \equiv 2,(1,0) \equiv 1) .
$$

So, the players' payoffs are

$$
v_{1}=1, v_{2}=2, v_{3}=2 . v_{1}=1, v_{2}=3, v_{3}=2 .
$$

$X^{*}$ is not equalized situation in $\Gamma(H)$.

\section{Applications}

In an organization of a teaching process of a subject at University we mean the functioning of the organizational $T$ system, that contains $S=\{1,2, \ldots, n\}$ group of students and $P$ - professor. Some individuals (players) who are having different interest take part in $T$ system. They canb choose one or seweral actions strategies. Choosing the relevant strategies finally they get some utilities. Players aim is to choose the optimal strategies, using them they will get the maximal utilities. Thus in mathematical model of the functioning $T$ system: players $\quad N=P \cup S=\{P, 1,2, \ldots, n\}$, sets of strategies of each player's $A_{i}(i=P, 1, \ldots, n)$ and players' interest expressing by $H_{i}(i=P, 1, \ldots, n)$ functions of utilities, should take part in.

It is clear that in $T$ system each player's behaviour may be focused on each player's utilities. Therefore according to our notes, $T$ system's functioning model is given by non-cooperative game

$$
\Gamma=<N,\left\{A_{i}\right\}_{i \in N},\left\{H_{i}\right\}_{i \in N}>.
$$

Let consider (10) model in the case of a situation at an exam. Where the number of players is two and where $i=1$ player is a student but $i=2$ player is a professor - $P$ or $N=\{1-s t ., 2-$ prof. $\}$, then (10) model represents a bimatrix game, Their sets of strategies are $A_{1}=\{1-$ to be ready properly, $2-$ not to be readly $\}$, $A_{2}=\{1-$ appraise positively, $2-$ not appraise positively\}. We have got four situations $(1,1),(1,2)$, $(2,1),(2,2)$. Let estimate them relatively with the following utilities $(2,0),(-1, .-2),(1,-3),(0,-1)$. Thus a bimatrix game has the following form

$$
\Gamma=\left(\begin{array}{cc}
(2,0) & (-1,-2) \\
(1,-3) & (0,-1)
\end{array}\right) .
$$

In this game $i=1$ chooses a line, $i=2$ - a column. There exists two equilibrium situations in pure strategies $X^{*}=(1,1), X^{* *}=(2,2)$ and the third equilibrium situation in mixed strategies

$$
X^{* * *}=(0,5 ; 0,5) \text {. }
$$

The question is how we can define (11) game in case of two or more players? For this firstly write (11) as a form of a $\Gamma(H)$ game, where a student and professor sit around the round table. According to (5) and (6) games' equivalency $\Gamma(H)$ game has the following for

$$
H_{1}=\left(\begin{array}{cc}
2 & -1 \\
1 & 0
\end{array}\right), H_{2}=\left(\begin{array}{cc}
0 & -3 \\
-2 & 1
\end{array}\right) .
$$

Here in $H_{1}$ player $i=1$ (student) chooses a line and $i=2$ (professor) chooses a column, but in $H_{2}$ player $i=2$ chooses a line and $i=1$ chooses a column. In $\Gamma(H)$ game $X^{*}=(1,1)$ and $X^{* *}=(2,2)$ are equilibrium situations in pure strategies. Analogically to the example 1 there exists the equilibrium situation and

$$
\begin{aligned}
& p_{1}=\frac{-1+3}{0-1+2+3}=\frac{1}{2}=0,5, \\
& p_{2}=\frac{0+1}{2+0-1+1}=\frac{1}{2}=0,5 .
\end{aligned}
$$

Therefore $X^{* * *}=(0,5 ; 0,5)$. Thus, (11) and (12) games have got one and the same equilibrium situations.

Now, in the situation of an exam for the purpose of students' optimal behaviour of a group $S=\{1,2, \ldots, n\}$ let them sit around the round table according to the given row and play (12) model so that one of the students sitting next to each other performs a role of a professor. In this case each of them will perform both roles as a student and as a professor as well.

Let consider three students' case $S=\{1,2,3\}$. Begin the play in case when $i=1$ is a student, $i=2$ - is a professor. Then

$$
\begin{gathered}
H_{1}=\left(\begin{array}{cc}
2 & -1 \\
1 & 0
\end{array}\right), H_{2}=\left(\begin{array}{cc}
0 & -3 \\
-2 & 1
\end{array}\right), \\
H_{3}=\left(\begin{array}{cc}
2 & -1 \\
1 & 0
\end{array}\right) .
\end{gathered}
$$

Analogically to 4-th and 5-th examples:

$$
\begin{gathered}
F\left(x_{1}\right)=(1-2) \cdot(0+1)=-1<0, \\
F\left(x_{2}\right)=(-2-0) \cdot(-1+3)<0, \\
F\left(x_{3}\right)=(1-2) \cdot(0+1)<0, \\
\Delta x_{1}, x_{2} H_{1}=2-1+1=2, \\
\Delta x_{2}, x_{3} H_{2}=-1+2+3=4, \\
\Delta x_{3}, x_{1} H_{3}=2+0-1+1=2, \\
\Delta x_{1} H_{1}(2)=0+1=1, \\
\Delta x_{2} H_{2}(2)=-1+3=2, \\
\Delta x_{3} H_{3}(2)=0+1=1,
\end{gathered}
$$




$$
\begin{gathered}
p_{1}=\frac{\Delta x_{3} H_{3}(2)}{\Delta x_{3}, x_{1} H_{3}}=0,5, \quad p_{2}=\frac{\Delta x_{1} H_{1}(2)}{\Delta x_{1}, x_{2} H_{1}}=0,5, \\
p_{3}=\frac{\Delta x_{2} H_{2}(2)}{\Delta x_{2}, x_{3} H_{2}}=\frac{2}{4}=0,5 .
\end{gathered}
$$

Hence $X^{*}=(0,5 ; 0,5 ; 0,5)$ is the equilibrium situation in (13) game. Besides, there exists the equilibrium situations in pure strategies

$$
X^{* *}=(1,1,1), X^{* * *}=(2,2,2) \text {. }
$$

In case of 4 students $\Gamma(H)$ game will have the following form:

$$
\begin{aligned}
& H_{1}=\left(\begin{array}{cc}
2 & -1 \\
1 & 0
\end{array}\right), H_{2}=\left(\begin{array}{cc}
0 & -3 \\
-2 & 1
\end{array}\right), \\
& H_{3}=\left(\begin{array}{cc}
2 & -1 \\
1 & 0
\end{array}\right), H_{4}=\left(\begin{array}{cc}
0 & -3 \\
-2 & 1
\end{array}\right) .
\end{aligned}
$$

There we'll find players' optimal strategies analogically to an example 3 game $\Gamma(H)$ will be defined the same way for any number of students. Overall, it is obvious that a student should study properly otherwise he will not get a positive grade.

\section{Conclusion}

The dyadic non-cooperative $\Gamma(H)$ game model which is discussed in this paper can be used in socialeconomic and political situations for making strategic decisions, where players share a unique set of strategies. The players in $\Gamma(H)$ game have to sit at a round table and each one has to coordinate each other in the values of utilities in each situation. According to this , we get the following players' payoff functions:

$$
\left.H_{1}\left(x_{1}, x_{2}\right), H\left(x_{2}, x_{3}\right), \ldots, H_{n}\left(x_{n}, x_{1}\right)\right) \text {, }
$$

where $i$-player's payoff, $i \in N$, depends on his own strategy and $i+1-$ player's strategies (taking $n+1 \equiv 1)$.

The term of an equalized situation is introduced for $\Gamma(H)$ game which is at the same time an equilibrium situation in $\Gamma(H)$ game. It is shown that in $\Gamma(H)$ game Nash's equilibrium situation in the mixed strategies is the equalized situation. The theorem gives the opportunity to find the criterium of the existence of an equilibrium situation of $\Gamma(H)$ game in the mixed strategies and its finding method. In $\Gamma(H)$ game it is possible to exist: 1 ) an equilibrium situation in the mixed and pure strategies; 2) an equilibrium situation only in mixed strategies; 3 ) an equilibrium situation only in pure strategies. In $\Gamma(H)$ game in the pure strategies in case of finding equilibrium situation, we are making the situations in the pure strategies and check each of them. In the case of two players $\Gamma(H)$ game is a $2 \times 2$ bimatrix $\Gamma$ game. The players' conditions of optimal mixed strategies existence in $\Gamma$ game is written.
Relevant examples are solved is and $\Gamma(H)$ game's application for finite amount of players' is discussed.

\section{References}

[1] Brams S. J. Game theory and politics. New York University, 2003,312 p.

[2] Brams S. J. Kilgour D. M. Game theory and national security. Basil Blackwell, New York, 1988, $199 \mathrm{p}$.

[3] Deemen A. Rusinowska. Editors. Collective Decision Making. Views from social choise and game theory. Springer Heidelberg Dordrecht London, New York, 2010, 281 p.

[4] Geckil I. K., Anderson P. Applied game theory and strategic behavior. Taylor and Francis Group, 2010, 212 p.

[5] Keet M. C. Terrorism and Game Theory. Coalitions, negotiations and audience costs. Limerick, University of Limerick, Ireland, July, 2003, 155 p.

[6] A. Kelly. Decision making using game theory. An introduction for managers. Cambridge University Press, 2003, 215 p.

[7] McCain R. Game theory and public policy. Drexel University, USA,2009, 270 p.

[8] McCarty N., A. Meirowitz Adam. Political game theory. An introduction. Cambridge University Press, 2007, 447 p.

[9] Ordeshook P. Game theory and political theory. An introduction. Cambridge University Press, 1986, $528 \mathrm{p}$.

[10] Rasmusen E. Games and information. An Introduction to game theory. Basil Blackwell, 2005, $577 \mathrm{p}$.

[11] Salikvadze M.E., G. N. Beltadze G.N., F. Criado. Dyadic theoretical games models of decision making for the lexicographic vector payoffs. International Journal of Information Technology and Decision Making, Vol. 8, Issue 2, 2009, pp. 193-216.

[12] Vorob'ev N.N. Foundations of game theory Noncooperative games. Birkhauser Verlag. Basel Boston - Berlin, 1994, 496 p.

G. N. Beltadze: Full Professor, 1982 - Ph.D. at St.Petersburg State University, 1992 - Dr. of Sci. of St.Petersburg State University. Fields of research: Game Theory, Operations Research.

J. A. Giorgobiani: Senior Scientist, 1965 - Ph.D. at Tbilisi State University. Fields of research: Game Theory, Operations Research. 